\title{
Matrix producing breast cancer with chondroid differentiation
}

\section{Quratulain Zulfiqar Ali, Lubna Mushtaque Vohra}

\begin{abstract}
Introduction: A type of Metaplastic Breast Cancer (MBC) that has matrix producing properties is an infrequently occurring breast cancer and is responsible for less than $1 \%$ of the overall cases. MBC is mixed in nature, comprising of a blend of adenocarcinomatous and metaplastic components. The occurrence of $\mathrm{MBC}$ with chondroid differentiation is rarer and also a diagnostic challenge; warranting its presentation. Case Report: A case of a 57-yearold female is reported here, having a $4.3 \times 3.3$ cm mass palpable at the lower outer quadrant of the right breast. Upon performing a trucut biopsy, an infiltrating malignant tumor was found, with focal chondroid differentiation in the background of a chondromyxoid matrix suggesting a metaplastic carcinoma. Conclusion: Early detection and treatment is of key significance as Metaplastic Breast Cancer is a rare entity with a histopathological variance, a higher rate of metastasis, locally recurrent and carry an overall poor prognosis.
\end{abstract}

Keywords: Breast cancer, Chondroid, Metaplastic, Public health, Women's health

Quratulain Zulfiqar Ali ${ }^{1}$, Lubna Mushtaque Vohra ${ }^{2}$

Affiliations: ${ }^{1}$ Postgraduate Resident Trainee, Internal Medicine, Ziauddin University Hospital, Karachi, Sindh, Pakistan; ${ }^{2}$ Assistant Professor, Department of Surgery and Oncology, Ziauddin University Hospital, Karachi, Sindh, Pakistan.

Corresponding Author: Quratulain Zulfiqar Ali, Flat Number 407, Adenwala Apartments, Britto Road, Garden East, Karachi, Sindh, Pakistan 74550; Email: quratulain_zulfiqar@ yahoo.com

Received: 30 April 2018

Accepted: 29 May 2018

Published: 23 June 2018

\section{How to cite this article}

Ali QZ, Vohra LM. Matrix producing breast cancer with chondroid differentiation. J Case Rep Images Oncology 2018;4:100053Z10QA2018.

Article ID: 100053Z10QA2018

$* * * * * * * * *$

doi: 10.5348/100053Z10QA2018CR

\section{INTRODUCTION}

Metaplastic Breast Cancer (MBC) is a subtype of breast tumors that is rare in its occurrence and diverse in its histological presentation; accounting for less than $1 \%$ of all breast cancers $[1,2]$. It is represented by the metaplastic transformation of the epithelium into Squamous or mesenchymal morphology [3]. Due to the rarity of $\mathrm{MBC}$ expressing the three hormone receptors, these tumors are labeled under the breast cancers that are triple negative (ER,PR and Her-2 negative) [4].

MBC with cartilaginous or osseous differentiation is an exceptional variety of breast cancers and its frequency is only $0.003-0.12 \%$ of the reported breast cancer cases [5]. A list of possible differential diagnosis was made; including malignant phyllodes tumor, malignant adenomyoepithelial tumor and $\mathrm{MBC}$ with chondrosarcoma.

\section{CASE REPORT}

A postmenopausal woman aged 57-year-old visited the clinic having an incidentally discovered right sided breast lump. On examination, the lump was situated in the lower outer quadrant of breast, approximately $4.5 \mathrm{~cm}$ in size, no skin retraction and impalpable lymph nodes in the axilla. She had a positive family history with two of 


\section{EDORIUM Journals}

www.edoriumjournals.com/case-reports/jcro

her sisters having had a Carcinoma of the breast in their postmenopausal age.

Ultrasound of Right breast showed a heterogeneous hypo echoic mass with irregular margins measuring $4.3 \times 3.3 \mathrm{~cm}$ at $5 / 6$ o clock position. Benign looking lymph nodes were seen in the right axilla. Mammography revealed an asymmetrical increased density mass with surrounding architectural distortion in the right breast's lower quadrant. It was categorized as BIRADS IV (Breast Imaging Reporting and Data System). She was subjected to core biopsy showing an infiltrating malignant tumor present in sheets and cords with focal chondroid differentiation and a chondromyxoid matrix (Figures 1, 2 and 3). On the basis of which she was diagnosed as having a metaplastic carcinoma with mesenchymal differentiation; grade III according to Modified Bloom Richardson grading system. CT Scan Chest and Whole Body Skeletal survey was negative for metastasis. She was staged according to AJCC (American Joint Committee on Cancer) $7^{\text {th }}$ version and clinically she was in Stage II of her disease.

She was offered neoadjuvant chemotherapy to downsize the tumor for breast conservation; however she refused chemotherapy and opted for mastectomy. She underwent a mastectomy with sentinel node biopsy. Histopathology demonstrated a $5.1 \times 3.3 \times 3 \mathrm{~cm}$ metaplastic tumor with matrix production properties, tumor necrosis and histological grade III. No evidence of lymphatic or perineural invasion, skin or nipple involvement and ductal or lobular carcinoma in situ (LCIS). A total of 5 lymph nodes were recovered all of which were tumor free (T3, No, Mo). The tumor was negative for ER (Estrogen Receptor), PR (Progesterone Receptor) and HER-2 (Human Epidermal Growth Factor Receptor 2) hormone receptors, negative for $\mathrm{p}-16$ with $15-20 \%$ expressivity for Ki-67.

She received adjuvant chemotherapy with $\mathrm{AC}$ x4 (Adriamycin and Cyclophosphamide) followed by 4 cycles of Taxane. Chemotherapy was followed by 21 sessions of right sided chest wall radiotherapy at a dose of 4500.0 cGy. Currently, she is doing well and regularly visiting the outpatient clinic for follow up.

\section{DISCUSSION}

The breast cancer incidence amongst women is increasing world wide and is one of the principal sources of deaths among women. Breast cancer has been reported to be the most commonly occurring cancer in Pakistani women, attributing for $34.6 \%$ of the cases [6]. This data is obtained from a sole cancer registry in Pakistan which is population based, named Karachi Cancer Registry. Due to the traditional and conservative nature of the society in the majority of the country, most of the breast cancers (metaplastic or otherwise) are reported at stages where salvage becomes challenging.

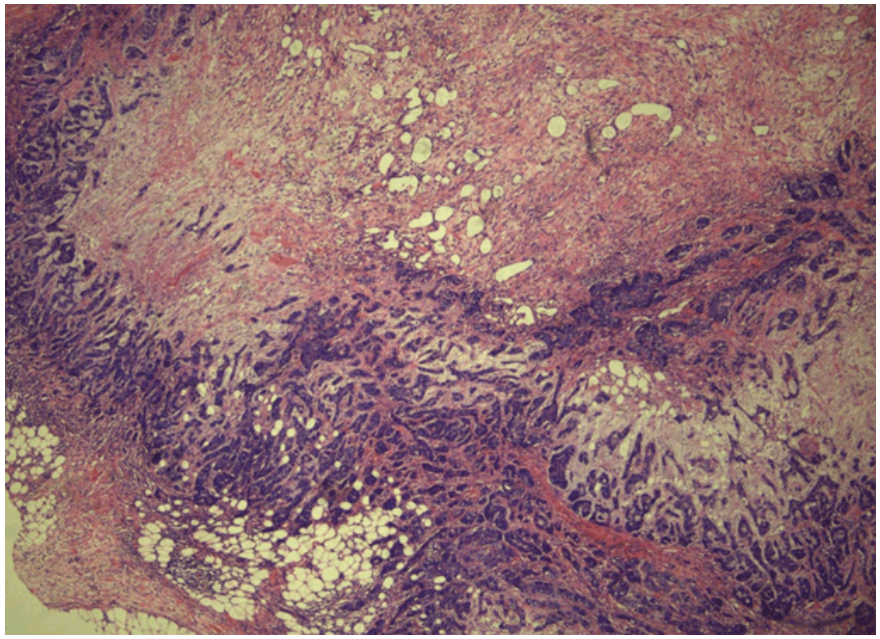

Figure 1: Section shows fibrocollagenous fragments with breast parenchyma exhibiting an infiltrating malignant tumor present in sheets and cords with focal chondroid differentiation and chondromyxoid matrix. Tumor cells are highly atypical with enlarged, irregular hyperchromatic nuclei, prominent nucleoli and pale eosinophilic cytoplasm. Haemotoxylin and Eosin stain used.

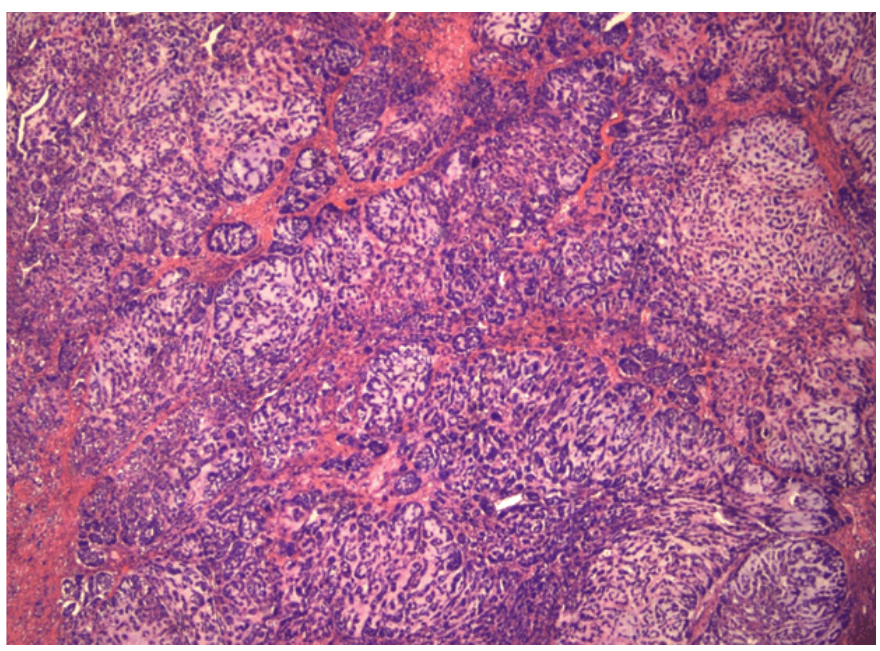

Figure 2: Metaplastic carcinoma with mesenchymal differentiation.

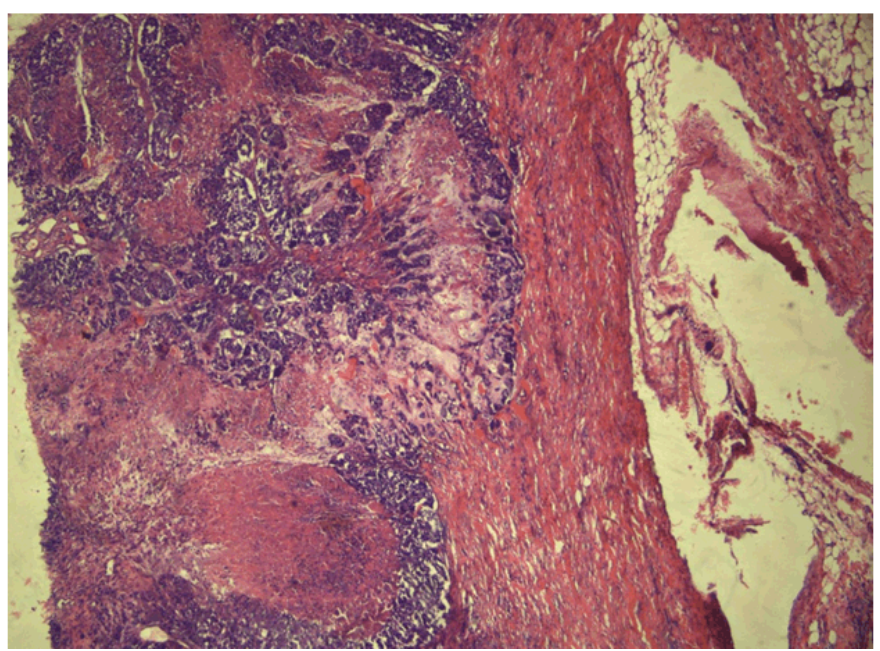

Figure 3: Malignant spindle cells with chondroid differentiation. 


\section{EDORIUM Journals}

www.edoriumjournals.com/case-reports/jcro

$\mathrm{MBC}$ is a subtype of breast cancers that is less than $1 \%$ in occurrence. According to the WHO guidelines, the classification of $\mathrm{MBC}$ is comprised of metaplastic carcinoma with fibromatosis, spindle cell carcinoma, low grade adenosquamous carcinoma, mesenchymal differentiated metaplastic carcinoma, mixed metaplastic carcinoma and myoepithelial carcinoma[7].

Pathogenesis of metaplastic breast cancer is still unclear. However, mutations in various genes such as oncogenes, tumor suppressor genes and proto-oncogenes play a vital role in cancer development [8]. BRCA1, BRCA2 and p53 mutations have a well-defined role as tumor suppressor genes in breast cancers [8]. Mutations in these tumor suppressor genes lead to loss of their inhibitory role on tumor growth resulting in unrestrained tumor progression. In high risk populations, these mutations can be diagnostically tested to predict the life time chance of breast cancer development according to the result.

MBC tumors are bulky, having a consistency of being firm to hard. Adherence to the underlying skin or deep tissues is also observed [9]. There is an increased likelihood for the tumor spread to be metastatic despite majority of MBC's not showing nodal involvement [10]. They tend to spread through hematological routes leading to their high rate of systemic metastasis; further highlighting the importance of early detection of MBC's [11]. Ultimate diagnosis is reached through a thorough histopathology analysis. As compared to the invasive ductal carcinomas (IDC), MBC has a higher proportion of triple negative cases with a comparatively higher expression of Ki-67 [12]. Furthermore, they are more likely to have local recurrences and have an overall poor prognosis [12].

Despite the knowledge that $\mathrm{MBC}$ is a separate entity, treatment regimens largely tend to follow the ones for Invasive Ductal Carcinoma (IDC)[13].

\section{CONCLUSION}

$\mathrm{MBC}$ is a rare entity with a histopathological variance. They are bulky tumors with a rare nodal involvement and a paradoxically higher rate of metastasis. Hence, further importance lies on early detection and treatment. Most of the cases of $\mathrm{MBC}$ are triple negative, they are highly likely to be locally recurrent and thus have a poor prognosis. There is no specific treatment for MBC per say and it mostly follows the treatment of IDC.

\section{REFERENCES}

1. Rosen PP. Carcinoma with metaplasia. In: Rosen PP, editor. Rosen's Breast Pathology. Philadelphia: Lippincott-Raven; 1997. p. 375-95.

2. Tavassoli FA. Classification of metaplastic carcinomas of the breast. Pathol Annu 1992;27 Pt 2:89-119.
3. Dieci MV, Orvieto E, Dominici M, Conte P, Guarneri V. Rare breast cancer subtypes: Histological, molecular, and clinical peculiarities. Oncologist 2014 Aug;19(8):805-13.

4. Lim KH, Oh DY, Chie EK, et al. Metaplastic breast carcinoma: Clinicopathologic features and prognostic value of triple negativity. Jpn J Clin Oncol 2010 Feb;40(2):112-8.

5. Tsukuda K, Tsuji H, Kunitomo T, et al. Breast cancer with cartilaginous and/or osseous metaplasia diagnosed by lymph nodal metastasis: A case report. Acta Med Okayama 2009 Dec;63(6):367-71.

6. Bhurgri Y. Karachi cancer registry data - Implications for the national cancer control program of Pakistan. Asian Pac J Cancer Prev 2004 Jan-Mar;5(1):77-82.

7. Lakhani SR, Ellis IO, Schnitt SJ, Tan PH, van de Vijver MJ. WHO Classification of Tumours of the Breast. 4ed. WHO Classification of Tumours, Volume 4. Lyon, France: International Agency for Research on Cancer; 2012.

8. Ostad SN, Parsa M. Breast cancer from molecular point of view: Pathogenesis and biomarkers. In: Gunduz M, Gunduz S, editors. Breast CancerFocusing Tumor Microenvironment, Stem cells and Metastasis. Rijeka, Croatia: InTech; 2011.

9. Sood S, Mahajan V, Kaushal V. Metaplastic carcinoma of the breast: A report of two cases along with review of literature. Clinical Cancer Investigation Journal 2014 Jul 1;3(4):335.

10. Rakha EA, Lee AH, Evans AJ, et al. Tubular carcinoma of the breast: Further evidence to support its excellent prognosis. J Clin Oncol 2010 Jan 1;28(1):99-104.

11. Tavassoli FA, Devilee P. World Helth Organiation Classification of Tumours: Pathology and Genetics of Tumours of the Breast and Female Genital Organs. Lyon, France: IARC Press; 2003.

12. SongY,LiuX,Zhang G, etal.Uniqueclinicopathological features of metaplastic breast carcinoma compared with invasive ductal carcinoma and poor prognostic indicators. World J Surg Oncol 2013 Jun 6;11:129.

13. Shah DR, Tseng WH, Martinez SR. Treatment options for metaplastic breast cancer. ISRN Oncol 2012;2012:706162.

$* * * * * * * * *$

\section{Author Contributions}

Quratulain Zulfiqar Ali - Conception and design, Acquisition of data, Drafting the article, Revising it critically for important intellectual content, Final approval of the version to be published

Lubna Mushtaque Vohra - Substantial contributions to conception and design, Acquisition of data, Analysis and interpretation of data, Drafting the article, Revising it critically for important intellectual content, Final approval of the version to be published

\section{Guarantor of Submission}

The corresponding author is the guarantor of submission.

\section{Source of Support}

None 


\section{Consent Statement}

Written informed consent was obtained from the patient for publication of this case report.

\section{Conflict of Interest}

Authors declare no conflict of interest.

\section{Copyright}

(C) 2018 Quratulain Zulfiqar Ali et al. This article is distributed under the terms of Creative Commons Attribution License which permits unrestricted use, distribution and reproduction in any medium provided the original author(s) and original publisher are properly credited. Please see the copyright policy on the journal website for more information.

\section{ABOUT THE AUTHORS}

Article citation: Ali QZ, Vohra LM. Matrix producing breast cancer with chondroid differentiation. J Case Rep Images Oncology 2018;4:100053Z10QA2018.

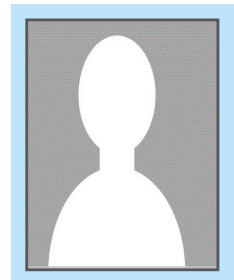

Quratulain Zulfiqar Ali has worked as an FCPS Postgraduate Resident Trainee at Ziauddin University Hospital in the Department of Internal Medicine. She has graduated from Dow University of Health Sciences, Karachi, Pakistan in the year 2012.

Email: Quratulain_zulfiqar@yahoo.com

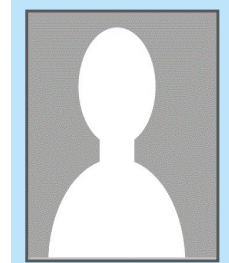

Lubna Mushtaque Vohra is working as an Assistant Professor (Consultant Breast Surgeon) at Ziauddin University Hospital. MBBS from Dow Medical College Karachi FCPS from CPSP Karachi, Pakistan. I have published seven research papers in national and international journals. My keen area of interest is Breast Diseases, Cancer. I aspire to pursue further research in Breast cancer genetic mutations in our population.

Email:Lubna_mushtaque@hotmail.com

Access full text article on other devices

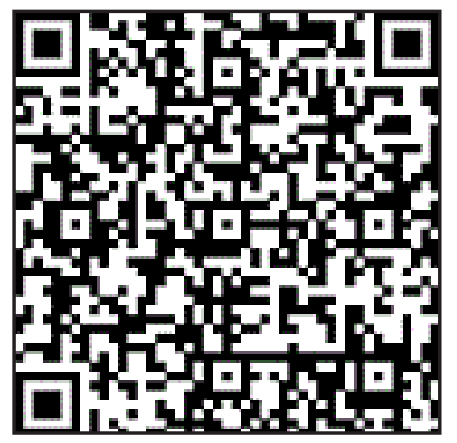

Access PDF of article on other devices

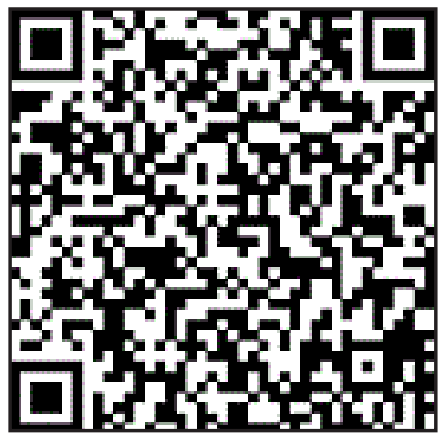

